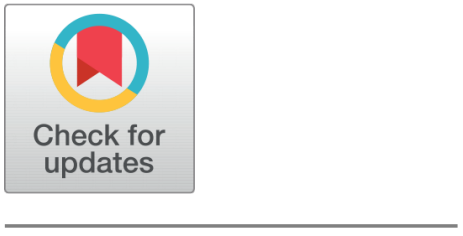

OPEN ACCESS

Received: 13.08 .2020

Accepted: 21.10 .2020

Published: 09.11.2020

Editor: Dr. Natarajan Gajendran

Citation: Arantza Jency A, Sharma RK, Singh G (2020)

Stationary solutions, critical mass, Tadpole orbits in the circular restricted three-body problem with the more massive primary as an oblate spheroid. Indian Journal of Science and Technology 13(39): 4168-4188. https://doi.org/ 10.17485/IJST/v13i39.1396

* Corresponding author.

Tel: +91-9566406224

arantzajen1997@gmail.com

Funding: None

Competing Interests: None

Copyright: (c) 2020 Arantza Jency et al. This is an open access article distributed under the terms of the Creative Commons Attribution License, which permits unrestricted use, distribution, and reproduction in any medium, provided the original author and source are credited.

Published By Indian Society for Education and Environment (iSee)

ISSN

Print: 0974-6846

Electronic: 0974-5645

\section{Stationary solutions, critical mass, Tadpole orbits in the circular restricted three-body problem with the more massive primary as an oblate spheroid}

\author{
A Arantza Jency ${ }^{1 *}$, Ram Krishan Sharma ${ }^{1}$, Gagandeep Singh ${ }^{1}$ \\ 1 Department of Aerospace Engineering, Karunya Institute of Technology and Sciences, \\ Coimbatore, 641114, Tamil Nadu, India. Tel.: +91-9566406224
}

\section{Abstract}

Background: The location and stability of the equilibrium points are studied for the Planar Circular Restricted Three-Body Problem where the more massive primary is an oblate spheroid. Methods: The mean motion of the equations of motion is formulated from the secular perturbations as derived by ${ }^{(1)}$ and used in ${ }^{(2-4)}$. The singularities of the equations of motion are found for locating the equilibrium points. Their stability is analysed using the linearized variational equations of motion at the equilibrium points. Findings: As the effect of oblateness in the mean motion expression increases, the location and stability of the equilibrium points are affected by the oblateness of the more massive primary. It is interesting to note that all the three collinear points move towards the more massive primary with oblateness. It is a new result. Among the shifts in the locations of the five equilibrium points, the $y$-location of the triangular equilibrium points relocate the most. It is very interesting to note that the eccentricities (e) of the orbits around $L_{1}$ and $L_{3}$ increase, while it decreases around $L_{2}$ with the addition of oblateness with the new mean motion. The decrease in e is significant in Saturn-Mimas system from 0.95036 to 0.87558 . Similarly, the value of the critical mass ratio $\mu_{c}$, which sets the limit for the linear stability of the triangular points, further reduces significantly from $0.285 \ldots A_{1}$ to $0.365 \ldots A_{1}$ with the new mean motion. The mean motion $\mathrm{s}_{z}$ in the z-direction increases significantly with the new mean motion from $9 A_{1} / 4$ to $9 A_{1} / 2$.

Keywords: Circular Restricted Three-Body Problem (CRTBP); oblateness; mean motion; equilibrium points; critical mass ratio; tadpole orbits

\section{Introduction}

The restricted three-body problem (RTBP) has played an essential role in many different areas of dynamical astronomy and this will continue to do so. The modern applications to space mechanics are more powerful if not more numerous than the classical applications. The implications of the restricted problem for cosmology and 
stellar dynamics are also numerous. Consider the famous classical three-body problem, the Sun-Earth combination and the determination of the motion of Moon. We might think of the two large bodies, the Sun and the Earth, which move around their centre of mass in approximate circles in their gravitational field and a third body, the Moon, moves on an approximate ellipse. This configuration is stationary in a sense, since no collisions take place. This is also true for the motions of a Trojan asteroid under the continued influence of Sun and Jupiter. On the other hand, one of the central problems in space science is to create artificial bodies. As such the study of the restricted three-body problem remained of utmost interest for many mathematicians and astronomers direct from Newton to Lagrange in the early days of its inception. In recent years, with the launching of artificial satellites in the Earth-Moon system and in the solar system, the applications of the restricted three-body problem to the celestial mechanics form the basis of some lunar and planetary theories. Euler was the first to contribute towards the restricted three-body problem in 1772 in connection with the lunar theories. Beginning with Euler and Lagrange in 1772, the theory of restricted three-body problem goes through Jacobi in 1836, Hill in1878, Brown in1896 and continue with Poincare in 1892, Levi-Civita in 1904 and Birkoff in 1915 and many more modern mathematicians and astronomers.

RTBP possesses five points of libration called equilibrium or Lagrangian points. Three collinear equilibrium points $\mathrm{L}_{1}, \mathrm{~L}_{2}$ and $\mathrm{L}_{3}$ lying on the line joining the two massive bodies are unstable in the Lyapunov sense, while two equilateral positions $\mathrm{L}_{4}$ and $\mathrm{L}_{5}$ are linearly stable for mass ratios $\mu<\mu_{0}=0.03852 \ldots$ The locations of the equilibrium points in the restricted three-body problem by assuming both the primaries as oblate spheroids with their equatorial planes coincident with the plane of motion was calculated in ${ }^{(5)}$. In ${ }^{(6)}$ the location of the collinear points in the same problem was studied numerically for some systems of astronomical interest. These equilibria were shown to be unstable in general, though the existence of conditional infinitesimal (linearized) periodic orbits around them was established. However, the secular effect of oblateness of the primaries on the motion of the primaries was not included. The oblateness of only the more massive primary was considered and the secular effect of oblateness ${ }^{(7)}$ on the mean motion of the primaries was included in ${ }^{(8-12)}$. In ${ }^{(8,9)}$, the critical mass value $\mu_{c}$ was found to decrease with oblateness. In ${ }^{(8,10)}$, a numerical investigation of the locations of the five equilibrium points was made for some systems of astronomical interest. Periodic solutions of the linearized equations of motion around the five equilibrium points were studied. The angular frequency in the $\mathrm{z}$-direction $\left(s_{z}\right)$ was found to be more than the mean motion $\mathrm{n}$. In ${ }^{(11)}$ it was established that the oblateness induces a one-to-one commensurability at the exterior point $L_{3}$ and at the interior point $L_{2}$ for 0 $\leq \mu \leq \frac{1}{2}$ and at $L_{1}$ no such commensurability exists. Series expansions were found for the long-periodic $\left(\mathrm{S}_{4}\right)$ short-periodic $\left(\mathrm{S}_{5}\right)$ orbits. $s_{4}$ was found to increase and $s_{5}$ was found to decrease with oblateness. A number of research publications were generated in RTBP by various authors by considering the more massive or smaller primary or both primaries as oblate spheroids with their equatorial planes coincident with the plane of motion. Some of the relevant publications are documented in ${ }^{(12-32)}$.

In this paper we have included the secular effects of oblateness on the mean anomaly, argument of perigee and right ascension of ascending node ${ }^{(1)}$. We have obtained a new expression for mean motion of the primaries. We have utilized the new mean motion to study the above problem. We have calculated the locations of the equilibrium points and studied the stability of the equilibrium points using linearized equations of motion. Some interesting conclusions are drawn.

The collinear points and the $y$-coordinate of the triangular equilibrium points shift nearer to the origin due to the new mean motion. But, the $x$-coordinate of the triangular points does not show a change due to the variation in the mean motion till linear terms of oblateness. As opposed to an increase in the eccentricity of the periodic orbits around the collinear points reported when the previous mean motion was used, the eccentricity at the collinear point $L_{2}$ decreases. The eccentricities of the orbits at the other equilibrium points increased earlier with oblateness and it increases further with the new mean motion. As the mean motion value increases, the critical mass value $\left(\mu_{c}\right)$ shows further decrease from the critical mass value $\left(\mu_{0}\right)$ of the unperturbed case. Both the angular frequencies of the triangular points increase until $\mu$ becomes 0.0266053866 . The value of mean motion at the triangular points in the $z$-direction shows further increase with the new mean motion.

The equations of motion, force function for the problem and the mean motion expression are defined (sections 1 and 2 ). The equilibrium points are located using the condition that the first derivative of the force function will equate to zero at these points (Section 3). The stability of the collinear equilibrium points are studied (Section 4.1). The critical mass ratio for the stability of the triangular equilibrium points is derived (Section 4.2). Also, the tadpole orbits around the triangular equilibrium points are studied (Section 5).

\section{Equations of Motion}

The problem is defined in the non-dimensional synodic coordinate frame as given by [ Figure 1 ]. The barycentre of the primaries mark the origin of the system which rotates about the $z$-axis (perpendicular to the plane of motion of primaries). The mass ratio $\mu$ is the ratio of the mass of less massive primary $m_{2}$ to the sum of the masses of the primaries $m_{1}+m_{2}$ which is unity in the non-dimensional system. Point $P$ represents the point mass (with infinitesimal mass). 
The equations of motion in terms of the dimensionless synodic frame is given by Equations $(1)^{(10,33)}$. The force function $\Omega^{(8,10)}$ in the equations is given by Equation (2). The oblateness of the more massive primary $\mathrm{A}_{1}=\left(\mathrm{R}_{E}^{2}-\mathrm{R}_{P}{ }^{2}\right) / 5 \mathrm{R}^{2}, \mathrm{R}_{E}$ and $\mathrm{R}_{P}$ are equatorial and polar radii, respectively, and $\mathrm{R}$ is the distance between the primaries, affects the force function (2) of the system.

$$
\begin{gathered}
x^{\prime \prime}-2 n y^{\prime}=\Omega_{x}, \\
y^{\prime \prime}+2 n x^{\prime}=\Omega_{y}, \\
\Omega=\frac{n^{2}}{2}\left((1-\mu) r_{1}^{2}+\mu r_{2}^{2}\right)+\left[\frac{1-\mu}{r_{1}}+\frac{\mu}{r_{2}}+\frac{(1-\mu) A_{1}}{2 r_{1}^{3}}\right] . \\
\Omega_{x}=\frac{\partial \Omega}{\partial x}=n^{2} x-\frac{(1-\mu)(x-\mu)}{r_{1}^{3}}-\frac{\mu(x+1-\mu)}{r_{2}^{3}}-\frac{3 A_{1}(x-\mu)(1-\mu)}{2 r_{1}^{5}}, \\
\Omega_{y}=\frac{\partial \Omega}{\partial y}=y\left[n^{2}-\frac{(1-\mu)}{r_{1}^{3}}-\frac{\mu}{r_{2}^{3}}-\frac{3 A_{1}}{2 r_{1}^{5}}\right] .
\end{gathered}
$$

Referring [ Figure 1], the distances $r_{1}$ and $r_{2}$ of $\mathrm{P}$ from the more massive and the smaller primaries are related to the distances $x$ and $y$ from the origin by

$$
\begin{aligned}
& r_{1}^{2}=(x-\mu)^{2}+y^{2}, \\
& r_{2}^{2}=(x+1-\mu)^{2}+y^{2}
\end{aligned}
$$

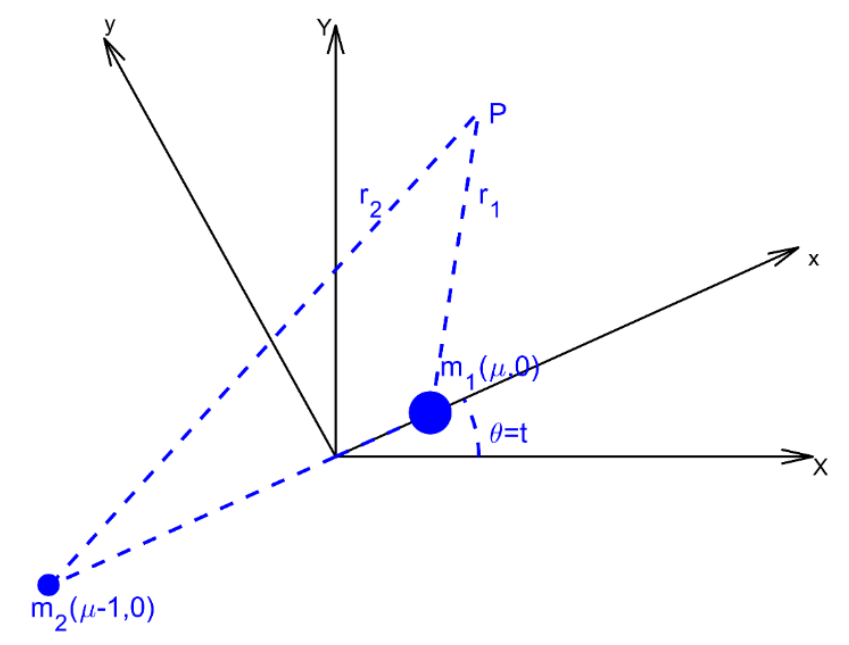

Fig 1. Planar Restricted Three Body Problem in the fixed (sidereal) and rotating (synodic) coordinate system.

\subsection{Mean Motion}

The mean motion equation (Equation 5) for this study is derived using the effect of perturbation $J_{2}$, given by Equations (4) ${ }^{(34)}$, on the three orbital elements - $M_{s}$ - mean anomaly, $\omega_{s}$ - argument of perigee and $\Omega_{s}$ - right ascension of the ascending 
node.

$$
\frac{d M_{s}}{d t}=n\left[1+\frac{3 J_{2}}{2 a^{2}\left(1-e^{2}\right)^{\frac{3}{2}}}\right], \frac{d \omega_{s}}{d t}=n\left[\frac{3 J_{2}}{a^{2}\left(1-e^{2}\right)^{2}}\right], \frac{d \Omega_{s}}{d t}=n\left[\frac{-3 J_{2}}{2 a^{2}\left(1-e^{2}\right)^{2}}\right],
$$

$\mathrm{J}_{2}$ is Earth second zonal harmonic; a and e are semi-major axis and eccentricity, respectively.

The mean motion $n$ is the summation of the changes in $M_{s}, \omega_{s}$ and $\Omega_{s}$ after one revolution ${ }^{(1)}$. Averaging the above equations for one revolution and summing them up, the mean motion expression after simplification is obtained, as in ${ }^{(1)}$, given by

$$
n=1+\frac{3 A_{1} R^{2}}{2 a^{2}\left[\left(1-e^{2}\right) R e\right]^{2}}\left(1+\sqrt{1-e^{2}}\right) .
$$

When the value of eccentricity e becomes zero and we get $\mathrm{n}=1+\frac{3 A_{1} R^{2}}{a^{2} R e^{2}}$, which upon non-dimensionalizing gives Equation

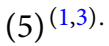

$$
n=1+3 A_{1}
$$

\section{Location of Equilibrium Points}

The equations of motion (1) are found to have singular solutions at the five points ${ }^{(33)}$ called the Lagrange points, liberation points or equilibrium points. Three of these equilibrium points (collinear equilibrium points $-L_{1}, L_{2}$ and $L_{3}$ ) lie on the line joining the primaries and the other two (triangular equilibrium points $-L_{4}$ and $L_{5}$ ) form nearly equilateral triangles ${ }^{(10)}$ with the primaries. At these equilibrium points the first derivatives of the force function are zero i.e., $\Omega_{x}=\Omega_{y}=0^{(10,33)}$.

\subsection{Location of Collinear Equilibrium Points}

As the collinear equilibrium points lie on the $x$-axis, in addition to the conditions $\Omega_{x}=\Omega_{y}=0$, they also satisfy $y=0$. Therefore, by equating $\Omega_{x}$ and $y$ to zero and making the corresponding substitutions from Equations (6), we get the seventh degree polynomials given by Equations (7), (8) and (9) for the locations of $L_{1}, L_{2}$ and $L_{3}$, respectively, which upon solving with the help of MATLAB for different values of $\mu$ and $A_{1}$ gives the locations of the collinear equilibrium points.

$$
\begin{gathered}
x_{1}=\mu-1-\rho_{1}, \\
x_{2}=\mu-1+\rho_{2}, \\
x_{3}=\mu+\rho_{3}, \\
y_{1}=y_{2}=y_{3}=0 . \\
\left(2+12 \mathrm{~A}_{1}\right) \rho_{1}^{7}+\left[\left(2+12 A_{1}\right)(5-\mu)\right] \rho_{1}^{6}+\left[\left(2+12 \mathrm{~A}_{1}\right)(10-4 \mu)\right] \rho_{1}^{5} \\
+\left[\left(2+12 \mathrm{~A}_{1}\right)(10-6 \mu)-2\right] \rho_{1}^{4}+\left[\left(2+12 \mathrm{~A}_{1}\right)(5-4 \mu)-4(1-\mu)\right] \rho_{1}^{3} \\
+\left[9 \mathrm{~A}_{1}(1-\mu)-12 \mu\right] \rho_{1}^{2}-8 \mu \rho_{1}-2 \mu=0, \\
\left(2+12 \mathrm{~A}_{1}\right) \rho_{2}^{7}-\left[\left(2+12 \mathrm{~A}_{1}\right)(5-\mu)\right] \rho_{2}^{6}+\left[\left(2+12 \mathrm{~A}_{1}\right)(10-4 \mu)\right] \rho_{2}^{5} \\
+\left[\left(2+12 \mathrm{~A}_{1}\right)(10-6 \mu)-(2-4 \mu)\right] \rho_{2}{ }^{4}+\left[\left(2+12 \mathrm{~A}_{1}\right)(5-4 \mu)-4+12 \mu\right] \rho_{2}^{3} \\
+\left[9 \mathrm{~A}_{1}(1-\mu)-12 \mu\right] \rho_{2}{ }^{2}+8 \mu \rho_{2}-2 \mu=0 \\
\\
\left(2+12 \mathrm{~A}_{1}\right) \rho_{3}^{7}+\left[\left(2+12 \mathrm{~A}_{1}\right)(2+\mu)\right] \rho_{3}{ }^{6}+\left[\left(2+12 \mathrm{~A}_{1}\right)(1+2 \mu)\right] \rho_{3}^{5} \\
+\left[\left(2+12 \mathrm{~A}_{1}\right) \mu-2\right] \rho_{3}{ }^{4}-4(1-\mu) \rho_{3}{ }^{3}-\left[\left(2+3 \mathrm{~A}_{1}\right)(1-\mu)\right] \rho_{3}^{2} \\
-6(1-\mu) \mathrm{A}_{1} \rho_{3}-3(1-\mu) \mathrm{A}_{1}=0 .
\end{gathered}
$$


The location and stability of the equilibrium points are analysed for the different planetary systems given in [ Table 1 ]. The locations of the three collinear equilibrium points for these systems with and without the oblateness coefficient $A_{1}$ and their corresponding shifts in kilometres are listed in Tables 2, 3 and 4 , respectively. The shifts in the position of the collinear equilibrium points are in a range of an order of $10^{1}$ to $10^{3}$ kilometres in the dimensional frame. It is interesting to note that all the three collinear points move towards the more massive primary with oblateness with the new mean motion taken in this study. Earlier in ${ }^{(10)}$, with the mean motion $n^{2}=1+3 A_{1} / 2$ being utilized so far in the literature, it was noticed that only $\mathrm{L}_{1}$ moves towards the barycentre. The present observation is very interesting with the new mean motion as the direction of shift of $\mathrm{L}_{2}$ and $\mathrm{L}_{3}$ has changed and symmetry is obtained in the shift of the collinear points. Maximum shift in Saturn - Tethys system of $226.3 \mathrm{~km}$ is observed. Maximum shift in Saturn - Mimas of $721.1 \mathrm{~km}$ is found. Maximum shift of $1151.8 \mathrm{~km}$ is found in $\mathrm{L}_{3}$ for Saturn-Mimas system, whose oblateness coefficient $\left(A_{1}\right)$ is maximum (0.004235) of the 11 systems chosen for the study.

Table 1. Planetary systems and their parameters

\begin{tabular}{lllll}
\hline S. No. & System & $\mu$ & $A_{1}$ & $\mathrm{R}(\mathrm{km})$ \\
\hline 1 & Jupiter - Io & 0.0000415283 & 0.0006701421 & 421800 \\
2 & Jupiter - Europa & 0.0000250794 & 0.0002646382 & 671100 \\
3 & Jupiter - Ganymede & 0.0000807835 & 0.0001040401 & 1070400 \\
4 & Jupiter - Callisto & 0.0000479677 & 0.0000337017 & 1882700 \\
5 & Saturn - Mimas & 0.0000000659 & 0.0042349996 & 185539 \\
6 & Saturn - Enceladus & 0.0000001480 & 0.0025865767 & 238037 \\
7 & Saturn - Tethys & 0.0000010950 & 0.0016835857 & 294672 \\
8 & Saturn - Dione & 0.0000020390 & 0.0010308526 & 377415 \\
9 & Saturn - Rhea & 0.0000032000 & 0.0005275432 & 527068 \\
10 & Saturn - Titan & 0.0002461294 & 0.0000981153 & 1221865 \\
11 & Saturn - Hyperion & 0.0000002000 & 0.0000667989 & 1500934 \\
\hline
\end{tabular}

Table 2. Locations of $L_{1}$

\begin{tabular}{llll}
\hline \multirow{2}{*}{ S. No. } & \multicolumn{3}{c}{$x_{L_{1}}$} \\
\cline { 2 - 4 } & with $A_{1}=0$ & with $A_{1} \neq 0$ & Shift $(\mathrm{km})$ \\
\hline 1 & -1.0241601645 & -1.0237986154 & 95.33237 \\
3 & -1.0204068383 & -1.0202647840 & 62.27845 \\
4 & -1.0301894364 & -1.0301312540 & 34.91631 \\
5 & -1.0253548320 & -1.0253362861 & 213.26510 \\
6 & -1.0028031696 & -1.0016537342 & 224.70248 \\
7 & -1.0036719149 & -1.0027279336 & 226.27425 \\
8 & -1.0071624574 & -1.0063945723 & 189.40271 \\
9 & -1.0088158731 & -1.0083140311 & 140.79427 \\
10 & -1.0102489352 & -1.0099818079 & 176.16102 \\
\hline
\end{tabular}


Table 3. Location of $L_{2}$

\begin{tabular}{llll}
\hline \multirow{2}{*}{ S. No. } & \multicolumn{3}{c}{$x_{L_{2}}$} \\
\cline { 2 - 4 } 1 & with $A_{1}=0$ & with $A_{1} \neq 0$ & Shift $(\mathrm{km})$ \\
2 & -0.9761410952 & -0.9758344587 & 129.33928 \\
3 & -0.9798175902 & -0.9796952645 & 82.09280 \\
4 & -0.9702478526 & -0.9702019533 & 49.13061 \\
5 & -0.9749723121 & -0.9749571393 & 28.56585 \\
6 & -0.9972019276 & -0.9933151479 & 721.14922 \\
7 & -0.9963367564 & -0.9945473470 & 425.94563 \\
8 & -0.9928694013 & -0.9919540167 & 269.73821 \\
9 & -0.9912315837 & -0.9907059755 & 198.37243 \\
10 & -0.9898142613 & -0.9895550788 & 136.60678 \\
11 & -0.9569373834 & -0.9568352642 & 124.77587 \\
\hline
\end{tabular}

Table 4. Location of $L_{3}$

\begin{tabular}{llll}
\hline \multirow{2}{*}{ S. No. } & \multicolumn{3}{c}{$x_{L_{3}}$} \\
\cline { 2 - 4 } & with $A_{1}=0$ & with $A_{1} \neq 0$ & Shift $(\mathrm{km})$ \\
\hline 1 & 1.0000173034 & 0.9990158024 & 422.43312 \\
3 & 1.0000104497 & 0.9996140741 & 266.00763 \\
4 & 1.0000336598 & 0.9998776948 & 166.94488 \\
5 & 1.0000199865 & 0.9999694445 & 95.15541 \\
6 & 1.0000000275 & 0.9937923687 & 1151.76281 \\
7 & 1.0000000617 & 0.9961746748 & 910.58362 \\
8 & 1.0000004562 & 0.9974982645 & 737.32583 \\
9 & 1.0000008496 & 0.9984632933 & 580.29681 \\
10 & 1.0000013333 & 0.9992123096 & 415.86916 \\
& 1.0001025539 & 0.9997339019 & 450.44300 \\
\hline
\end{tabular}

\subsection{Location of Triangular Equilibrium Points}

Solving the equations $\Omega_{x}=0$ and $\Omega_{y}=0$, the values of $r_{1}$ and $r_{2}$ are related to the mean motion values by the following equations.

$$
\begin{aligned}
& 2 r_{1}^{5} n^{2}-2 r_{1}^{2}-3 A_{1}=0, \\
& r_{2}=\eta^{-\frac{2}{3}}
\end{aligned}
$$

Substituting the mean motion value $\mathrm{n}$ and solving Equations (10) by series expansion method, we get the values of $r_{1}$ and $r_{2}$. The Equations (11) are obtained by truncating the higher-order terms (of orders 2 and above) of oblateness coefficient $A_{1}$. While the value of $r_{1}$ remains undisturbed by the oblateness of the more-massive primary in the CRTBP in the literature ${ }^{(10,14)}$, withthe new mean motion, both the values of $r_{1}$ and $r_{2}$ are affected by $A_{1}$. Also, the effect of $A_{1}$ increases in the case of $r_{2}$.

$$
\begin{aligned}
& r_{1}=1-\frac{3}{2} A_{1}, \\
& r_{2}=1-2 A_{1} .
\end{aligned}
$$

Substituting these values in Equations (3) and solving for $x$ and $y$, the triangular equilibrium points are located by Equations (12). These points form near-equilateral triangles ${ }^{(8,10)}$ with the primaries. Equations (12) suggest that the varied mean motion 
does not affect the $x$-location of the triangular equilibrium points ${ }^{(14)}$ till linear terms of $A_{1}$, but it affects the $y$-location of $\mathrm{L}_{4,5}$. However, the effect of new mean motion is noticed in the $x$-coordinate of the triangular points when higher order terms are taken into account. Also, when the necessary changes are made from elliptic RTBP to the CRTBP, the equations show excellent agreement with ${ }^{(3)}$. Also, ${ }^{(3)}$ shows that the effect of $A_{1}$ on the location of the triangular equilibrium points is reduced by the orbital eccentricity of the primaries.

$$
\begin{aligned}
& x=\mu-\frac{1}{2}-\frac{A_{1}}{2} \\
& y= \pm \frac{\sqrt{3}}{2}\left(1-\frac{7}{3} A_{1}\right) .
\end{aligned}
$$

The locations of the triangular equilibrium points for the systems in [ Table 1] and their shifts due to the oblateness effect are given in [ Table 5]. [ Table 5] shows that the triangular equilibrium points are shifted away from the barycentre in the $x$-direction and that they move towards the barycentre in the $y$-direction. Among the shifts in the location of the equilibrium points, the shift in the $y$-direction of the triangular points are the maximum. They range above 200 kilometres in the dimensional coordinates for all the cases taken in [ Table 1]. Maximum shifts of $392.9 \mathrm{~km}$ and $1587.8 \mathrm{~km}$ in the $\mathrm{x}$ and y co-ordinates, respectively, are noticed in the Saturn-Mimas system, whose oblateness coefficient $A_{1}$ is maximum of the 11 systems chosen for this study.

\begin{tabular}{|c|c|c|c|c|c|c|}
\hline \multirow{2}{*}{ S. No. } & \multicolumn{3}{|c|}{$x_{L_{4}, L_{5}}$} & \multicolumn{3}{|c|}{$y_{L_{4}, L_{5}}$} \\
\hline & $A_{1}=0$ & $A_{1} \neq 0$ & Shift $(\mathrm{km})$ & $A_{1}=0$ & $A_{1} \neq 0$ & Shift (km) \\
\hline 1 & -0.4999584717 & -0.5002935428 & 141.33297 & \multirow{11}{*}{ \pm 0.8660254038} & \pm 0.8646712303 & 571.19039 \\
\hline 2 & -0.4999749206 & -0.5001072397 & 88.79935 & & \pm 0.8654906425 & 358.87829 \\
\hline 3 & -0.4999192165 & -0.4999712366 & 55.68226 & & \pm 0.8658151673 & 225.03718 \\
\hline 4 & -0.4999520323 & -0.4999688832 & 31.72509 & & \pm 0.8659573019 & 128.21545 \\
\hline 5 & -0.4999999341 & -0.5021174339 & 392.87879 & & \pm 0.8574676302 & 1587.80075 \\
\hline 6 & -0.4999998520 & -0.5012931404 & 307.85048 & & \pm 0.8607986411 & 1244.16289 \\
\hline 7 & -0.4999989050 & -0.5008406979 & 248.05278 & & \pm 0.8626233385 & 1002.49339 \\
\hline 8 & -0.4999979610 & -0.5005133873 & 194.52962 & & \pm 0.8639423332 & 786.18209 \\
\hline 9 & -0.4999968000 & -0.5002605716 & 139.02557 & & \pm 0.8649593836 & 561.86515 \\
\hline 10 & -0.4997538706 & -0.4998029283 & 59.94183 & & \pm 0.8658271397 & 242.25200 \\
\hline 11 & -0.4999998000 & -0.5000331995 & 50.13037 & & \pm 0.8658904215 & 202.59948 \\
\hline
\end{tabular}

Table 5. Locations of the triangular equilibrium points

\section{Stability of Equilibrium Points}

Representing the location of equilibrium points by $L_{i}(a, b)$, the motion near the equilibrium points can be approximated using the equations $x=a+\xi$ and $y=b+\eta^{(8,10) \text {. The }}$ variational equations of motion for $(\xi, \eta)$ in the linear analysis from Equations (1) are obtained as

$$
\begin{aligned}
& \xi^{\prime \prime}-2 n \eta^{\prime}=\Omega_{x x}(a, b) \xi+\Omega_{x y}(a, b) \eta \\
& \eta^{\prime \prime}+2 n \xi^{\prime}=\Omega_{x y}(a, b) \xi+\Omega_{y y}(a, b) \eta .
\end{aligned}
$$

Its characteristic equation and general solution are given by Equations (14) and (16), respectively ${ }^{(8,10)}$. These equations are used to study the linear stability of the equilibrium points. The points are said to be stable if any disturbance is nullified over time without causing a permanent change in the location of the points ${ }^{(18)}$.

$$
\begin{gathered}
\lambda^{4}+\left(\sigma_{1}\right) \lambda^{2}+\left(\sigma_{2}\right)=0, \\
\Lambda^{2}+\left(\sigma_{1}\right) \Lambda+\left(\sigma_{2}\right)=0, \\
\sigma_{1}=\left(4 n^{2}-\Omega_{x x}-\Omega_{y y}\right) \\
\sigma_{2}=\left(\Omega_{x x} \Omega_{y y}-\Omega_{x y}^{2}\right),
\end{gathered}
$$




$$
\begin{gathered}
\xi=\sum_{i=1}^{4} \alpha_{i} e^{\lambda_{i} t}, \\
\eta=\sum_{i=1}^{4} \beta_{i} e^{\lambda_{i} t} .
\end{gathered}
$$

\subsection{Stability of Collinear Equilibrium Points}

At the collinear points, we have

$$
\begin{aligned}
& \Omega_{x x}=n^{2}+\frac{2(1-\mu)}{r_{1}^{3}}+\frac{2 \mu}{r_{2}^{3}}+\frac{6(1-\mu) A_{1}}{r_{1}^{5}}>0, \\
& \Omega_{x y}=0, \\
& \Omega_{y y}=n^{2}-\frac{(1-\mu)}{r_{1}^{3}}-\frac{\mu}{r_{2}^{3}}-\frac{3 A_{1}(1-\mu)}{2 r_{1}^{5}} .
\end{aligned}
$$

It can be seen that $\Omega_{x x}>0, \Omega_{x y}=0, \Omega_{y y}<0$. This results in $\sigma_{2}<0$. The roots of Equations (13) are

$$
\begin{aligned}
& \lambda_{1,2}= \pm v_{1}= \pm \sqrt{\frac{-\sigma_{1}+\sqrt{\sigma_{1}^{2}-4 \sigma_{2}}}{2}} \\
& \lambda_{3,4}= \pm i v_{2}= \pm \sqrt{\frac{-\sigma_{1}-\sqrt{\sigma_{1}^{2}-4 \sigma_{2}}}{2}} .
\end{aligned}
$$

where, the first two roots are real and the other two are imaginary. This proves that the collinear points are unstable ${ }^{(10,13,15)}$. But, if the initial conditions $\left(\xi_{0}, \eta_{0}\right)$ of Equations (16) are chosen such that the values of $\alpha_{1}, \alpha_{2}$ are zero, we can generate periodic elliptic orbits around the collinear equilibrium points ${ }^{(10)}$. These elliptic orbits are centred around the collinear points with their semi-major and semi-minor axes parallel to the $y$ - and $x$-axes, respectively. The orbit's eccentricity $e_{o}$ and semi-major axis $a_{o}$ are given by Equations $(18)^{(1,10) \text {. }}$

$$
e_{o}=\sqrt{1-\frac{1}{\sigma_{3}^{2}}}, a_{o}=\sqrt{\sigma_{3}^{2} a^{2}+b^{2}}
$$

where

$$
\sigma_{3}=\frac{v_{2}^{2}+\Omega_{x x}}{2 v_{2}} .
$$

[ Table 6 ] lists the eccentricity of the periodic orbits around $L_{1}, L_{2}$ and $L_{3}$ for the systems in [ Table 1 ]. The eccentricities of the system are affected by the oblateness of the more massive primary. Eccentricities of the orbits around $L_{1}$ and $L_{3}$ increase with the addition of oblateness ${ }^{(10)}$, whereas the eccentricities of the orbits around $L_{2}$ decrease due to the change in the mean motion instead of an increase observed in ${ }^{(10)}$. There is significant decrease in eccentricity for the orbit around $\mathrm{L}_{2}$ in Saturn-Mimas system from 0.95036 to 0.87558 . This is again proved by the plots Figures 2,3 and 4 . To show the effect of $A_{1}$ on the eccentricity $e_{o}$, the orbits around $L_{1}, L_{2}$ and $L_{3}$ in the non-dimensional frame for the Saturn-Mimas system (with highest $A_{1}$ value) in [ Table 1] are plotted in Figures 2, 3 and 4 respectively, with an unchanged semi-major axis value. 
Table 6. Eccentricities of the periodic orbits near the collinear Lagrangian points

\begin{tabular}{lllllll}
\hline & \multicolumn{3}{c}{ Eccentricity } \\
\cline { 2 - 7 } S.No. & \multicolumn{3}{c}{$\mathrm{L}_{1}$} & $\mathrm{~L}_{2}$ & \multicolumn{2}{c}{$\mathrm{L}_{3}$} \\
\cline { 2 - 7 } & $A_{1}=0$ & $A_{1} \neq 0$ & $A_{1}=0$ & $A_{1} \neq 0$ & $A_{1}=0$ & $A_{1} \neq 0$ \\
\hline 1 & 0.9485726180 & 0.949983158 & 0.9517748579 & 0.9504026696 & 0.8660254044 & 0.866315809 \\
2 & 0.9488207988 & 0.949483051 & 0.9515277322 & 0.9508874119 & 0.8660254040 & 0.866140029 \\
3 & 0.9481740739 & 0.948353168 & 0.9521709192 & 0.9520031906 & 0.8660254059 & 0.866070457 \\
4 & 0.9484936280 & 0.948562395 & 0.9518534081 & 0.9517884343 & 0.8660254045 & 0.866039997 \\
5 & 0.9499881584 & 0.985790165 & 0.9503617692 & 0.8755885755 & 0.8660254038 & 0.867869261 \\
6 & 0.9499303335 & 0.974203452 & 0.9504195923 & 0.9073909887 & 0.8660254038 & 0.867149122 \\
7 & 0.9496983011 & 0.960152007 & 0.9506516499 & 0.9377346474 & 0.8660254038 & 0.866755971 \\
8 & 0.9495885513 & 0.955148141 & 0.9507614127 & 0.9446457582 & 0.8660254038 & 0.866472355 \\
9 & 0.9494935059 & 0.952034416 & 0.9508564709 & 0.9482440412 & 0.8660254038 & 0.866253987 \\
10 & 0.9472712881 & 0.947602961 & 0.9530631126 & 0.9528248103 & 0.8660254238 & 0.866068146 \\
11 & 0.9499045071 & 0.950725478 & 0.9504454252 & 0.9496186425 & 0.8660254038 & 0.866054331 \\
\hline
\end{tabular}

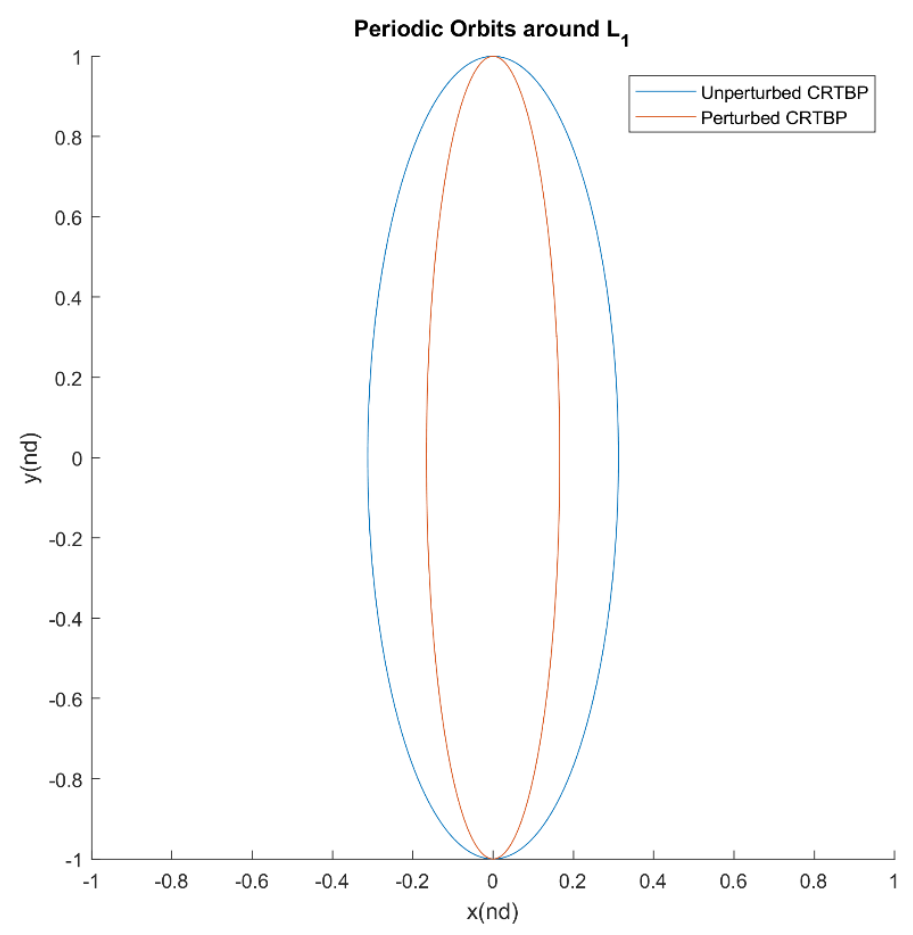

Fig 2. Periodic orbits around the collinear equilibrium point $L_{1}$ 


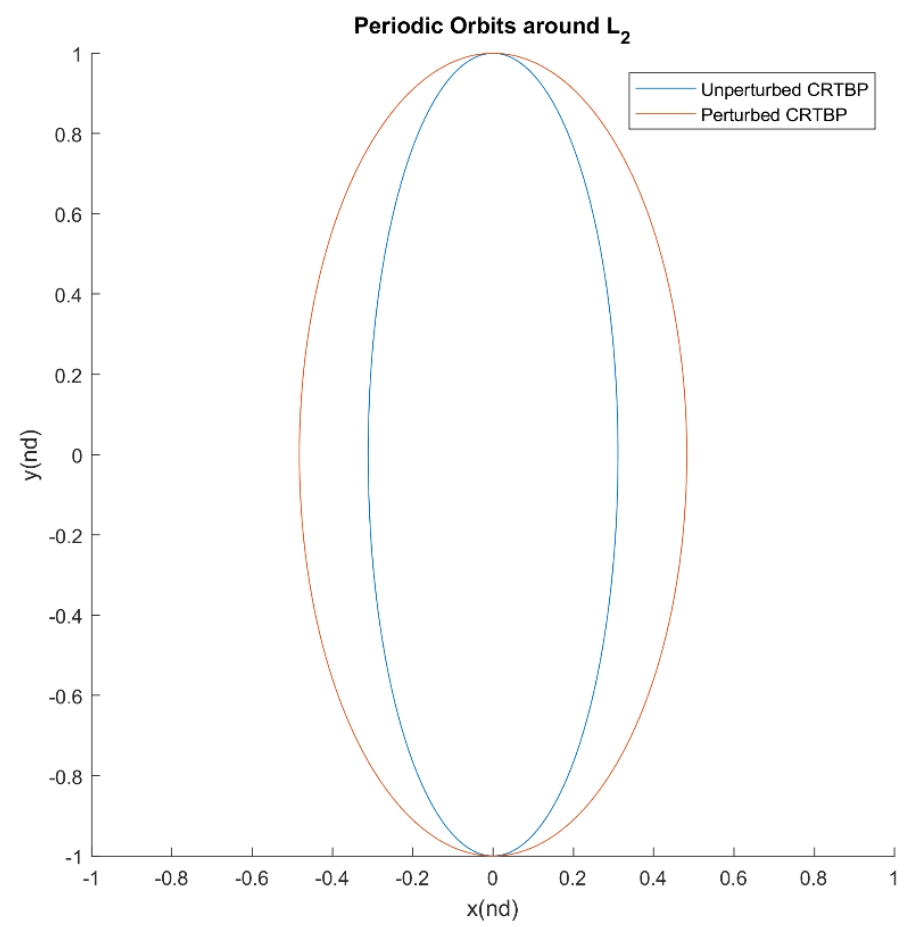

Fig 3. Periodic orbits around the collinear equilibrium point $L_{2}$

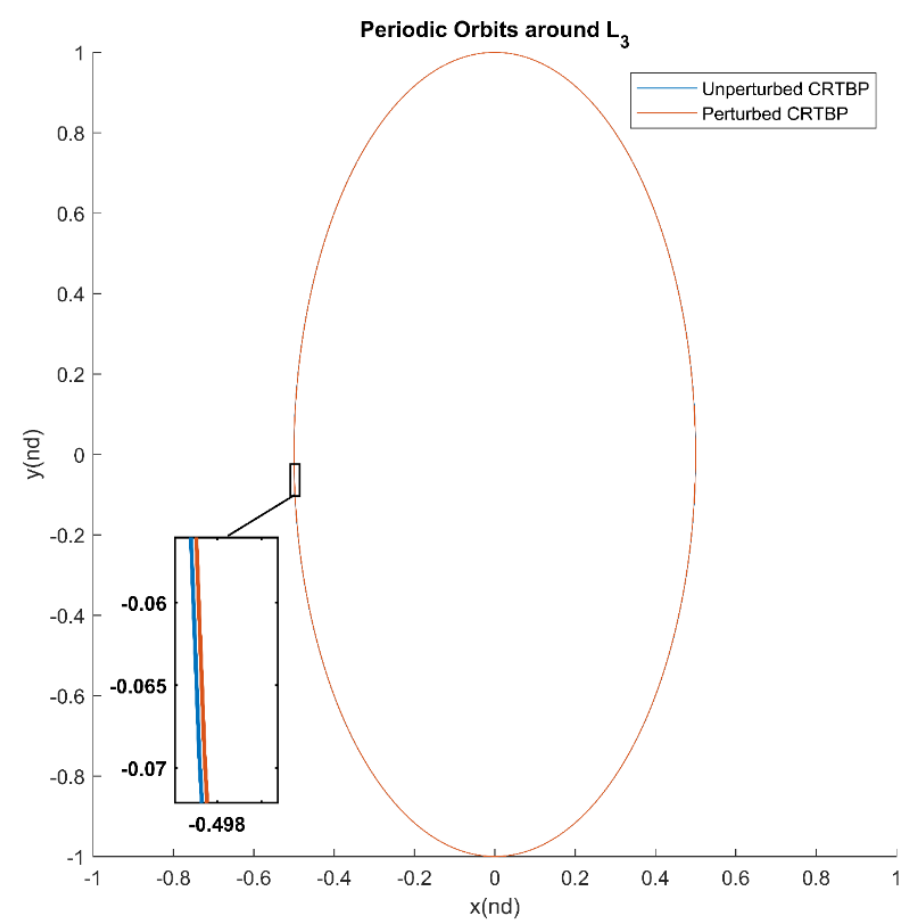

Fig 4. Periodic orbits around collinear equilibrium point $L_{3}$ 


\subsection{Stability of Triangular Equilibrium Points}

\subsubsection{Critical mass ratio}

At the triangular points $L_{4}$ and $L_{5}$, we have

$$
\begin{aligned}
& \Omega_{x x}=(x-\mu) f+(x+1-\mu) g>0, \\
& \Omega_{x y}= \pm y[f(x-\mu)+g(x+1-\mu)], \\
& \Omega_{y y}=y^{2}(f+g)>0,
\end{aligned}
$$

where

$$
f=3(1-\mu) / r_{1}^{5}+15 A_{1} / 2 r_{1}^{7}>0, g=3 \mu / r_{2}^{5}>0 .
$$

Substituting the values of $r_{1}$ and $r_{2}$ in the above equations from (11) at $\mathrm{L}_{4}$, we get

$$
\begin{aligned}
& \Omega_{x x}=\frac{3}{4}+9 A_{1}-3 \mu A_{1}, \\
& \Omega_{y y}=\frac{9}{4}+12 A_{1}, \\
& \Omega_{x y}=\frac{\sqrt{3}}{2}\left(3 \mu-\frac{3}{2}+23 \mu A_{1}-13 A_{1}\right) .
\end{aligned}
$$

The roots of the characteristic Equations (14) are

$$
\Lambda_{1,2}=\sqrt{\frac{-\sigma_{1} \pm \sqrt{\sigma_{1}^{2}-4 \sigma_{2}}}{2}} .
$$

where, $D=\sigma_{1}^{2}-4 \sigma_{2}$. As given by ${ }^{(33)}$, for the triangular equilibrium points to be stable, the discriminant $\mathrm{D}$ of Equation (14) should be zero. Therefore, $\sigma_{1}^{2}-4 \sigma_{2}=0$. Applying this condition results in Equation (22) which enables in finding a limiting value for the mass ratio, called the critical mass ratio $\mu_{c}$, within which the system will be linearly stable.

$$
1-6 A_{1}+\left(-27-84 A_{1}\right) \mu_{c}+\left(27+90 A_{1}\right) \mu_{c}^{2}=0 .
$$

Solving Equation (22) and truncating the higher order terms of $A_{1}$, the critical mass ratio is

$$
\begin{gathered}
\mu_{c}=\frac{1}{2}\left(1-\frac{\sqrt{69}}{9}\right)-\frac{1}{9}\left(1+\frac{19}{\sqrt{69}}\right) A_{1}, \\
\mu_{c}=\mu_{0}-0.3652590232 A_{1} .
\end{gathered}
$$

With $\mathrm{n}^{2}=1+3 \mathrm{~A}_{1} / 2$, we have from ${ }^{(8)}$,

$$
\begin{gathered}
\mu_{c}=\frac{1}{2}\left(1-\frac{\sqrt{69}}{9}\right)-\frac{1}{9}\left(1+\frac{13}{\sqrt{69}}\right) A_{1}, \\
\mu_{c}=\mu_{0}-0.28500179 A_{1} .
\end{gathered}
$$

Here $\mu_{0}$ is the critical mass ratio for the unperturbed CRTBP which is equal to $0.038520896505^{(33)}$. From equations (24) and (26) we prove that the critical mass ratio $\mu_{c}$ further decreases with the new mean motion value. It is inferred from ${ }^{(4)}$ that the value of $\mu_{c}$ increases with the oblateness of the smaller primary. It may be pointed out that the critical mass ratio $\mu_{c}$ in Equations (23) and (24) agrees as a particular case with that of ${ }^{(3)}$, after making appropriate changes to convert the ERTBP to CRTBP.

From Equations (23) to (26), it can be seen that with the coefficient of $A_{1}$ increase in the new mean motion expression, the critical mass ratio $\mu_{c}$ further decreases ${ }^{(8,14)}$, where the mean motion value is $n^{2}=1+3 \frac{A 1}{2}$. [ Table 7 ] lists the critical mass ratio values for different oblateness coefficients from ${ }^{(8)}$ and with Equation (23), which clearly depicts further reduction in the critical mass ratio $\mu_{c}$ with the current mean motion. 
Table 7. Variation of critical mass ratio with $A_{1}$

\begin{tabular}{lll}
\hline$A_{1}$ & $\mu_{c}{ }^{(8)}$ & $\mu_{c}$ \\
\hline 0.100000 & 0.010020717725 & 0.001994994186 \\
0.050000 & 0.024270807115 & 0.020257945345 \\
0.010000 & 0.035670878627 & 0.034868306273 \\
0.005000 & 0.037095887866 & 0.036694601389 \\
0.001000 & 0.038235894717 & 0.038155637481 \\
0.000500 & 0.038378395611 & 0.038338266993 \\
0.000100 & 0.038492396326 & 0.038484370602 \\
0.000050 & 0.038506646415 & 0.038502633553 \\
0.000010 & 0.038518046487 & 0.038517243914 \\
0.000005 & 0.038519471496 & 0.038519070209 \\
0.000001 & 0.038520611503 & 0.038520531246 \\
\hline
\end{tabular}

\subsubsection{Long- and short-periodic terms when the discriminant is greater than zero}

When $0<\mu \leq \mu_{c}$, the discriminant $D$ of Equation (21) is greater than zero. This gives a linearly stable solution of the characteristic equation for the triangular points with long- and short-periodic terms ${ }^{(10,11)}$ given by Equations (27).

$$
\begin{aligned}
& \lambda_{1,2}= \pm i\left(-\Lambda_{1}\right) \frac{1}{2}= \pm i s_{4} \\
& \lambda_{3,4}= \pm i\left(-\Lambda_{2}\right)^{\frac{1}{2}}= \pm i s_{5}
\end{aligned}
$$

The series expansions restricting to $\mathrm{A}_{1}$ terms of $s_{4}$ and $s_{5}$ with the oblateness of the more massive primary are:

$$
\begin{aligned}
s_{4}=\frac{3 \sqrt{3} \sqrt{\mu}}{2}[1 & +\frac{23}{8} \mu+\frac{4439}{128} \mu^{2}+\frac{548711}{1024} \mu^{3}+O\left(\mu^{4}\right) \\
& \left.+A_{1}\left(\frac{37}{6}+\frac{2291}{48} \mu+\frac{680627}{768} \mu^{2}+\frac{114994403}{6144} \mu^{3}+O\left(\mu^{4}\right)\right)\right] \\
s_{5}=1-\frac{27}{8} \mu- & \frac{3213}{128} \mu^{2}-\frac{355023}{1024} \mu^{3}+O\left(\mu^{4}\right) \\
& -A_{1}\left(-\frac{3}{2}+\frac{561}{16} \mu+\frac{133767}{256} \mu^{2}+\frac{21453741}{2048} \mu^{3}+O\left(\mu^{4}\right)\right)
\end{aligned}
$$

\begin{tabular}{|c|c|c|c|c|c|}
\hline \multirow{2}{*}{ S. No. } & \multicolumn{2}{|c|}{$s_{4}$} & \multicolumn{2}{|c|}{$s_{5}$} & \multirow[b]{2}{*}{$s_{z}$} \\
\hline & $A_{1}=0$ & $A_{1} \neq 0$ & $A_{1}=0$ & $A_{1} \neq 0$ & \\
\hline 1 & 0.0167446409 & 0.0168435533 & 0.9998597987 & 1.0008630298 & 1.0030110645 \\
\hline 2 & 0.0130119318 & 0.0130391763 & 0.9999153412 & 1.0003119086 & 1.0011901537 \\
\hline 3 & 0.0233568446 & 0.0233723479 & 0.9997271917 & 0.9998829326 & 1.0004680583 \\
\hline 4 & 0.0179964257 & 0.0180002369 & 0.9998380512 & 0.9998885445 & 1.0001516437 \\
\hline 5 & 0.0006669521 & 0.0063495951 & 0.9999997776 & 1.0063124178 & 1.0188792841 \\
\hline 6 & 0.0009995003 & 0.0039960385 & 0.9999995005 & 1.0038644146 & 1.0115726317 \\
\hline 7 & 0.0027186939 & 0.0037268803 & 0.9999963043 & 1.0025152732 & 1.0075476494 \\
\hline 8 & 0.0037099072 & 0.0040399936 & 0.9999931183 & 1.0015369402 & 1.0046281238 \\
\hline
\end{tabular}

From Equations (28), it is obvious that the long-period frequency $\left(s_{4}\right)$ increases with oblateness and a further increase is observed when comparing the series expansion of $s_{4}$ with that of ${ }^{(11)}$. Similarly, truncating the higher order terms, the shortperiod frequency $\left(s_{5}\right)$ decreases with $A_{1}$ when $\mu>0.0266053866$. The variation of $s_{4}$ and $s_{5}$ with $A_{1}$ are tabulated in [ Table 8].

Table 8. Angular Frequencies - $L_{4,5}$ 


\begin{tabular}{lrllll}
\hline \multicolumn{2}{c}{ Table 8 continued } & \multicolumn{5}{l}{} \\
\hline 9 & 0.0046476228 & 0.0047292641 & 0.9999891997 & 1.0007798303 & 1.0023711307 \\
10 & 0.0407888777 & 0.0408138380 & 0.9991677874 & 0.9993140892 & 1.0004413852 \\
11 & 0.0011618957 & 0.0011666839 & 0.9999993250 & 1.0000995128 & 1.0003005499 \\
\hline
\end{tabular}

This solution results in a retrograde periodic elliptic motion ${ }^{(12)}$ which is inclined at an angle $\alpha$, a function of the second derivatives. From ${ }^{(12)}$, the relation between $\alpha$ and the second derivatives is given by

$$
\alpha=\frac{1}{2} \tan ^{-1}\left[\frac{2 \Omega_{x y}}{\Omega_{x x}-\Omega_{y y}}\right]
$$

When the mean motion is $n^{2}=1+\frac{3}{2} A_{1}$,

$$
\tan 2 \alpha=\sqrt{3}-2 \sqrt{3} \mu+A_{1}\left(\frac{8}{\sqrt{3}}-\frac{16}{\sqrt{3}} \mu+4 \sqrt{3} \mu^{2}\right)
$$

When the mean motion is $n^{2}=1+6 A_{1}$ (the current study),

$$
\tan 2 \alpha=\sqrt{3}-2 \sqrt{3} \mu+A_{1}\left(\frac{20}{\sqrt{3}}-\frac{40}{\sqrt{3}} \mu+4 \sqrt{3} \mu^{2}\right)
$$

It is clearly seen from Equations (30) and (31) that the angle $\alpha$ increases with $A_{1}$ and that this increase is further enhanced with the inclusion of the new mean motion expression.

\subsubsection{Secular solutions when the discriminant is equal to zero}

When the discriminant is negative, the roots of Equations (14) will give a complex value with non-zero real and imaginary parts resulting in an unstable solution. But, we get purely imaginary roots [Equations (32)] when the discriminant $D=0$.

$$
\begin{gathered}
\lambda_{1,3}=i k \frac{1}{2}, \quad \lambda_{2,4}=-i k \frac{1}{2} \\
k=\frac{\sigma_{1}}{2}>0 .
\end{gathered}
$$

However, elliptic retrograde orbits can be established if the initial coordinates are combined with special velocity components. Transforming the coordinates at the triangular points about an angle $\alpha$ given by (29):

$$
\begin{aligned}
& \xi=\bar{\xi}_{\cos \alpha-\bar{\eta} \sin \alpha} \\
& \eta=\bar{\xi} \sin \alpha+\bar{\eta} \cos \alpha
\end{aligned}
$$

Substituting the above equation in Equations (13), we get

$$
\begin{gathered}
\bar{\xi}^{\prime \prime}-2 n \bar{\eta}=\lambda_{2}^{*} \bar{\xi}, \\
\eta^{\prime \prime}+2 n \bar{\xi}=\lambda_{1}^{*} \bar{\eta},
\end{gathered}
$$

where

$$
\lambda_{1,2}^{*}=\left(2 n^{2}-k\right) \pm 2 n \sqrt{n^{2}-k}
$$


Solving Equations (34), using Laplace transformation, the solutions can be written ${ }^{(12)}$ in terms of the initial position and velocities $\left(\bar{\xi}_{0}, \bar{\eta}_{0}, \bar{\xi}_{0}^{\prime}, \bar{\eta}_{0}^{\prime}\right)$ as

$$
\begin{aligned}
& \bar{\xi}=\left[\bar{\xi}_{0}+\left\{\frac{\left(k+\overline{\lambda_{1}^{*}}\right) \bar{\xi}_{0}^{\prime}}{2 k}-\frac{n \bar{\lambda}_{1}^{*} \overline{\eta_{0}}}{k}\right\} t\right] \cos \sqrt{k} t \\
& +\left[\left\{\frac{n \overline{\lambda_{1}^{*} \bar{\eta}_{0}}}{k \frac{3}{2}-\frac{\left(\bar{\lambda}_{1}^{*}-k\right) \bar{\xi}_{0}^{\prime}}{2 k \frac{3}{2}}}\right\}+\left\{\frac{\left(k+\overline{\lambda_{2}^{*}}\right) \overline{\xi_{0}}}{2 k \overline{\frac{1}{2}}+\frac{n \bar{\eta}_{0}^{\prime}}{\frac{1}{2}}}\right\} t\right] \sin \sqrt{k} t,
\end{aligned}
$$

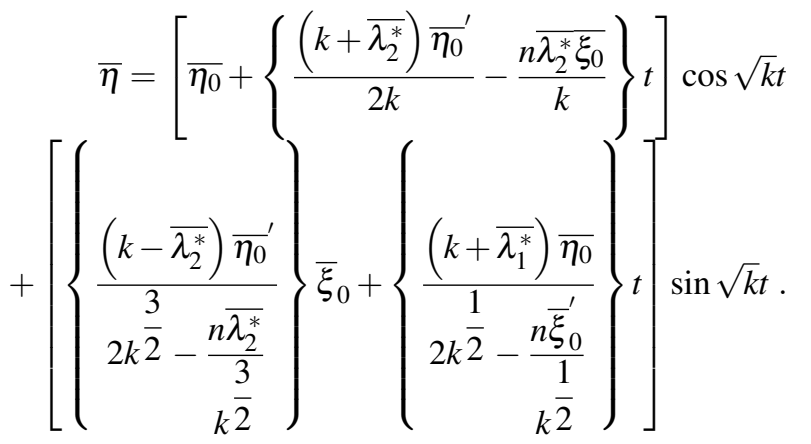

It is clear that, in this case also, due to the secular terms in $t$, the solution is unstable ${ }^{(12)}$. However, retrograde elliptic periodic orbits can be shown when the initial conditions are combined with special velocity components as

$$
\bar{\xi}_{0}^{\prime}=\frac{\sqrt{k} \bar{\eta}_{0}}{m}, \bar{\eta}_{0}^{\prime}=-\sqrt{k} m \bar{\xi}_{0} .
$$

where

$$
m=\sqrt{\frac{\left(k+\lambda_{2}^{*}\right)}{\left(k+\lambda_{1}^{*}\right)}} .
$$

Eliminating the secular terms from Equations (37), we get

$$
\begin{aligned}
& \bar{\xi}=\bar{\xi}_{0} \cos \sqrt{k} t+\frac{\bar{\eta}_{0}}{m} \sin \sqrt{k} t \\
& \bar{\eta}=\bar{\eta}_{0} \cos \sqrt{k} t-m \bar{\xi}_{0} \sin \sqrt{k} t
\end{aligned}
$$

Further eliminating $t$ from the above equations, we have

$$
m^{2} \bar{\xi}^{2}+\bar{\eta}^{2}=m^{2} \bar{\xi}_{0}^{2}+{\overline{\eta_{0}}}^{2}
$$

Equations (41) represents an ellipse with eccentricity $e=\sqrt{1-m^{2}}$, which after some algebra is equal to

$$
e=\sqrt{2(\sqrt{2}-1)}\left[1-\frac{3(2-\sqrt{2})}{4} A_{1}(\mu-1)\right]
$$


It can be seen that the eccentricity exactly matches with the result of ${ }^{(12)}$ till the first power of $A_{1}$. However, we have noticed that when the higher order terms of $A_{1}$ are included, the eccentricity shows a further increase. At $\left(\bar{\xi}_{0}, 0\right)$, the velocity components from Equations (38) become

$$
\bar{\xi}_{0}^{\prime}=0, \bar{\eta}_{0}^{\prime}=-\sqrt{k} m \bar{\xi}_{0}
$$

As $\sqrt{k} m$ is always positive, $\bar{\eta}_{0}$ always gets a sign opposite to that of $\bar{\xi}_{0}$. This shows that the periodic orbits represented by Equations (40) are retrograde.

\section{Tadpole Orbits around Triangular Equilibrium points}

For small distances from the triangular equilibrium points, trajectories in the synodic frame can be obtained by integrating the variational equations of motion. This way, we obtain particle paths, a combination of the short- and long-periodic orbits called the Tadpole orbits ${ }^{(35)}$ starting very close to the triangular points. These tadpole orbits define the movement of Trojan bodies around $L_{4}$ and $L_{5}$ points of the Sun-Jupiter system ${ }^{(35)}$. The Trojan orbits can be approximated by using the Tadpole orbits with suitable perturbations in the Restricted Three-Body Problem ${ }^{(36)}$.

With the help of the second derivatives of the force function $\left(\Omega_{x x}, \Omega_{x y}, \Omega_{y y}\right)$ at the triangular equilibrium points, the roots of Equations (14) are expressed as

$$
\begin{aligned}
& \lambda_{1,2}= \pm i v_{1}= \pm \sqrt{\frac{-\sigma_{1}+\sqrt{\sigma_{1}^{2}-4 \sigma_{2}}}{2}}, \\
& \lambda_{3,4}= \pm i v_{2}= \pm \sqrt{\frac{-\sigma_{1}-\sqrt{\sigma_{1}^{2}-4 \sigma_{2}}}{2}}
\end{aligned}
$$

Here, all the four roots of the system are found to be imaginary, if the value of the mass ratio $\mu$ of the system lies within the range $0<\mu<\mu_{c}{ }^{(33)}$. This, in turn makes the equilibrium points $L_{4}$ and $L_{5}$ stable ${ }^{(33,37)}$. In Equations (44), $v_{1,2}$ are the frequencies of the long- and short-periodic orbits. As all of the roots are proved to be imaginary, we can write the solution of Equations (14) to be

$$
\begin{aligned}
& \xi(f)=\alpha_{1} \cos \left(v_{1} f\right)+\alpha_{2} \sin \left(v_{1} f\right)+\alpha_{3} \cos \left(v_{2} f\right)+\alpha_{4} \sin \left(v_{2} f\right) \\
& \eta(f)=\beta_{1} \cos \left(v_{1} f\right)+\beta_{2} \sin \left(v_{1} f\right)+\beta_{3} \cos \left(v_{2} f\right)+\beta_{4} \sin \left(v_{2} f\right)
\end{aligned}
$$

We have chosen the Saturn-Titan system $\left(\mu=0.0002461294, A_{1}=0.0000981153\right)$ to examine the Tadpole orbits under the CRTBP with the oblateness of the more massive primary and to understand the effect of $A_{1}$ on the tadpole orbits. The initial conditions $^{(21)}$ of Equations (45) are $\xi_{0}=\xi(0)=\alpha_{1}+\alpha_{3}, \xi_{0}^{\prime}=\xi^{\prime}(0)=\alpha_{4} v_{2}+\alpha_{2} v_{1}, \eta_{0}=\eta(0)=\beta_{1}+\beta_{3}, \eta_{0}^{\prime}=\eta^{\prime}(0)=$ $\beta_{4} v_{2}+\beta_{2} v_{1}$. The coefficients are related to each other ${ }^{(21)}$ as:

$$
\begin{aligned}
& \alpha_{1}=-\frac{2 \beta_{2} v_{1}+\Omega_{x y} \beta_{1}}{v_{1}^{2}+\Omega_{x x}}, \quad \alpha_{2}=\frac{2 \beta_{1} v_{1}-\Omega_{x y} \beta_{2}}{v_{1}^{2}+\Omega_{x x}} \\
& \alpha_{3}=\frac{-2 \beta_{4} v_{2}-\Omega_{x y} \beta_{3}}{v_{2}^{2}+\Omega_{x x}}, \quad \alpha_{4}=\frac{2 \beta_{3} v_{2}-\Omega_{x y} \beta_{4}}{v_{2}^{2}+\Omega_{x x}} \\
& \beta_{1}=\frac{2 \alpha_{2} v_{1}-x y \alpha_{1}}{v_{1}^{2}+\Omega_{y y}}, \quad \beta_{2}=-\frac{2 \alpha_{1} v_{1}+\Omega_{x y} \alpha_{2}}{v_{1}^{2}+\Omega_{y y}}
\end{aligned}
$$




$$
\beta_{3}=\frac{2 \alpha_{4} v_{2}-\Omega_{x y} \alpha_{3}}{v_{2}^{2}+\Omega_{y y}}, \quad \beta_{4}=-\frac{2 \alpha_{3} v_{2}+\Omega_{x y} \alpha_{4}}{v_{2}^{2}+\Omega_{y y}}
$$

The initial conditions for the current case (sample orbits) are chosen to be $\xi_{0}=0.0001, \xi_{0}^{\prime}=0.005, \eta_{0}=0.0001, \eta^{\prime}(0)=0.005$ and the system of equations are solved using MATLAB to get the coefficients $\alpha_{1}, \alpha_{2}, \alpha_{3}, \alpha_{4}$ and $\beta_{1}, \beta_{2}, \beta_{3}, \beta_{4}$ of the Equations (45). Among these coefficients, $\alpha_{1}, \alpha_{2}, \beta_{1}, \beta_{2}$ correspond to the long-periodic orbit and $\alpha_{3}, \alpha_{4}, \beta_{3}, \beta_{4}$ correspond to the shortperiodic orbit.

[ Figure 6 ] shows the long- and short-periodic orbits of the Saturn-Titan System. The periodic eccentricity and the semimajor axis of the long- and short-periodic orbits can be calculated by using the Equations (18) and (19). The eccentricity and the semi-major axis of the long-periodic orbit are 0.9943196 and $9.448440 \mathrm{e}-04$, respectively in the non-dimensional frame when $A_{1}=0$. When $A_{1}$ is included, both the values increase and become 0.99432100 and $9.4495728 \mathrm{e}-04$, respectively. Considering the short-periodic orbit, the eccentricity decreases from 0.5535525 to 0.5533317 and the semi-major axis increases from $1.3287025 \mathrm{e}-04$ to $1.3287564 \mathrm{e}-04$ by taking $A_{1}$ into account. [ Figure 5 ] gives the combined long- and short-period, the Tadpole orbit plotted for the Saturn-Titan system for 400 Earth days. The tadpole orbit is affected by $A_{1}$ and shows a shift of up to 100 $\mathrm{km}$ in its trajectory in the dimensional frame.

Figures 7 and 8 shows the frequencies of the Tadpole orbits. It is clearly seen that the values locally repeat with a time period given by the short-period over a mean line which tends to vary with a frequency of the long-period. The orbital frequency of the long- and short-periodic orbits are 0.0399984 and 0.9991997 , respectively, in the unperturbed CRTBP. When oblateness effect is included, the frequency of the long-periodic orbit increases to 0.0400031 and that of the short-periodic orbit decreases to 0.9991400 . Therefore, when $A_{1} \neq 0$, time period of the long-periodic orbit $\left(T_{1}\right)$ decreases and that of the short periodic orbit $\left(T_{2}\right)$ increases. In the synodic system, the orbital period of the long- and short-periodic orbits are 157.0676 and 6.2886, respectively. The orbital period of Titan around Saturn is 15.945 Earth days. Taking that into account and converting the synodic system into dimensional coordinates, the long-period of the Tadpole orbit is 398.5944 Earth days and the short-period is 15.9587 Earth days.

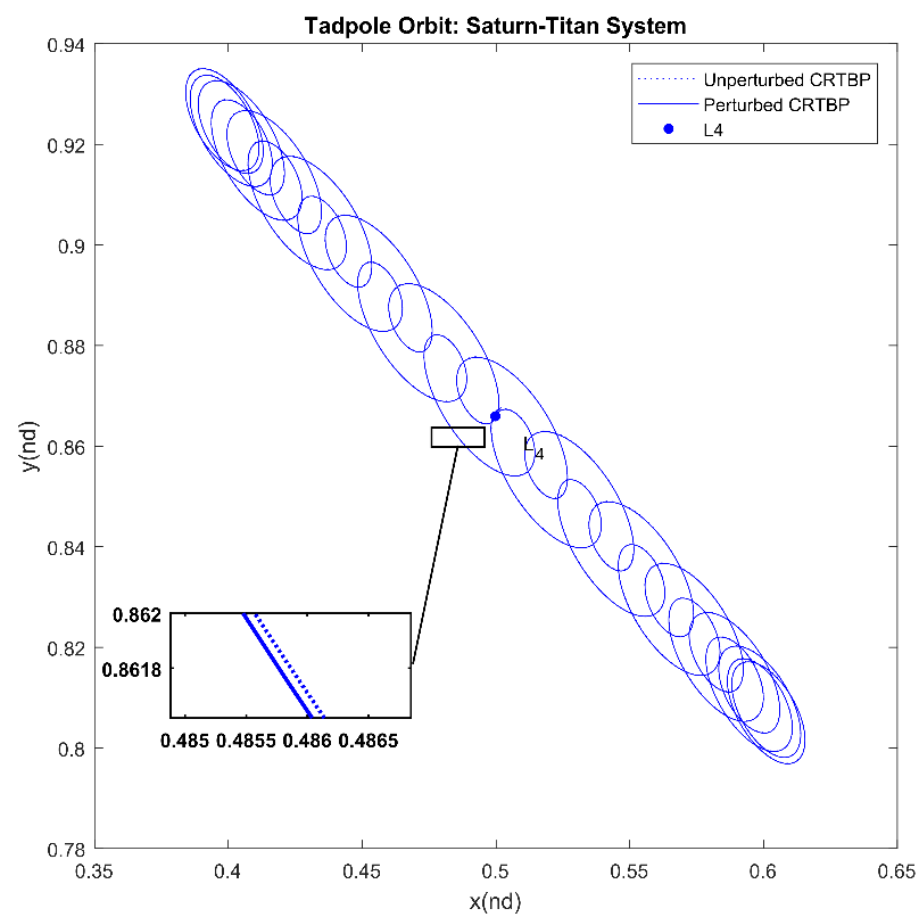

Fig 5. Tadpole orbit around triangular equilibrium point $L_{4}$ in Saturn-Titan System for 400 Earth days 


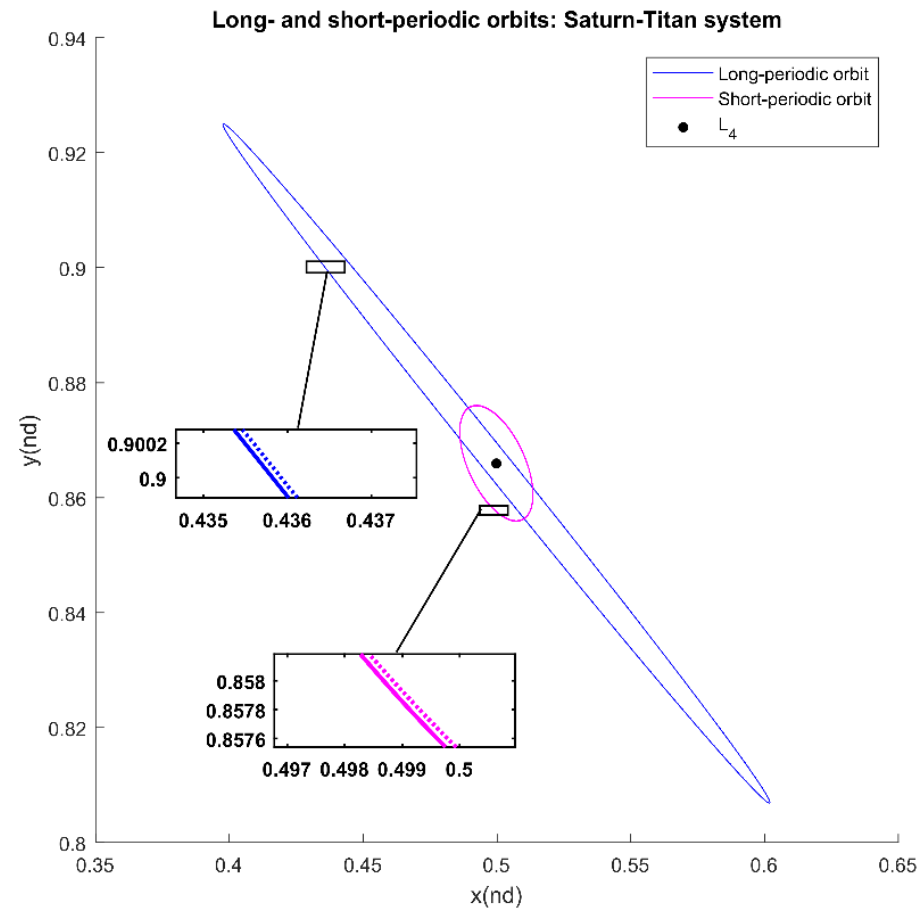

Fig 6. Long- and short-periodic orbits of Tadpole orbits (Saturn-Titan system)

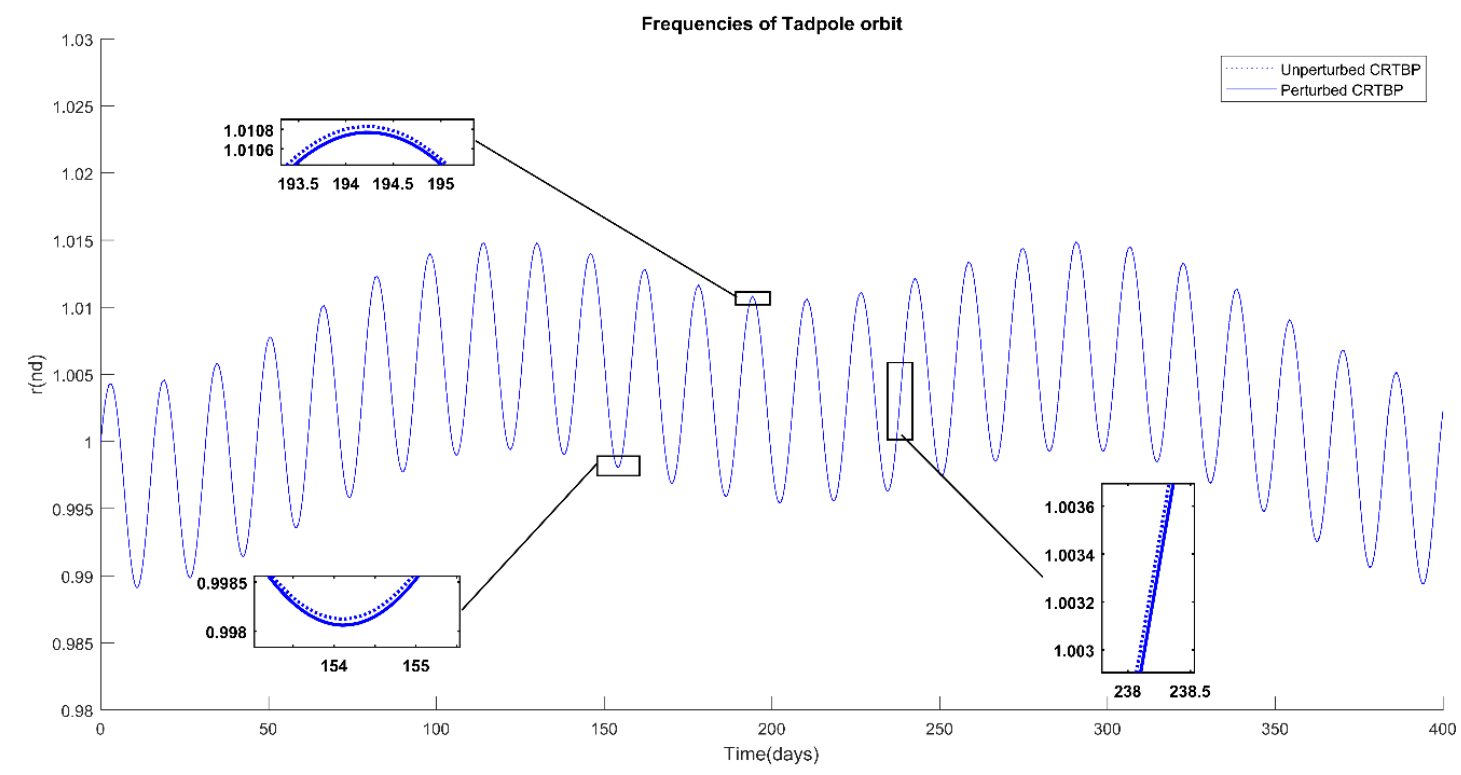

Fig 7. Frequencies of the Tadpole Orbits (position) 


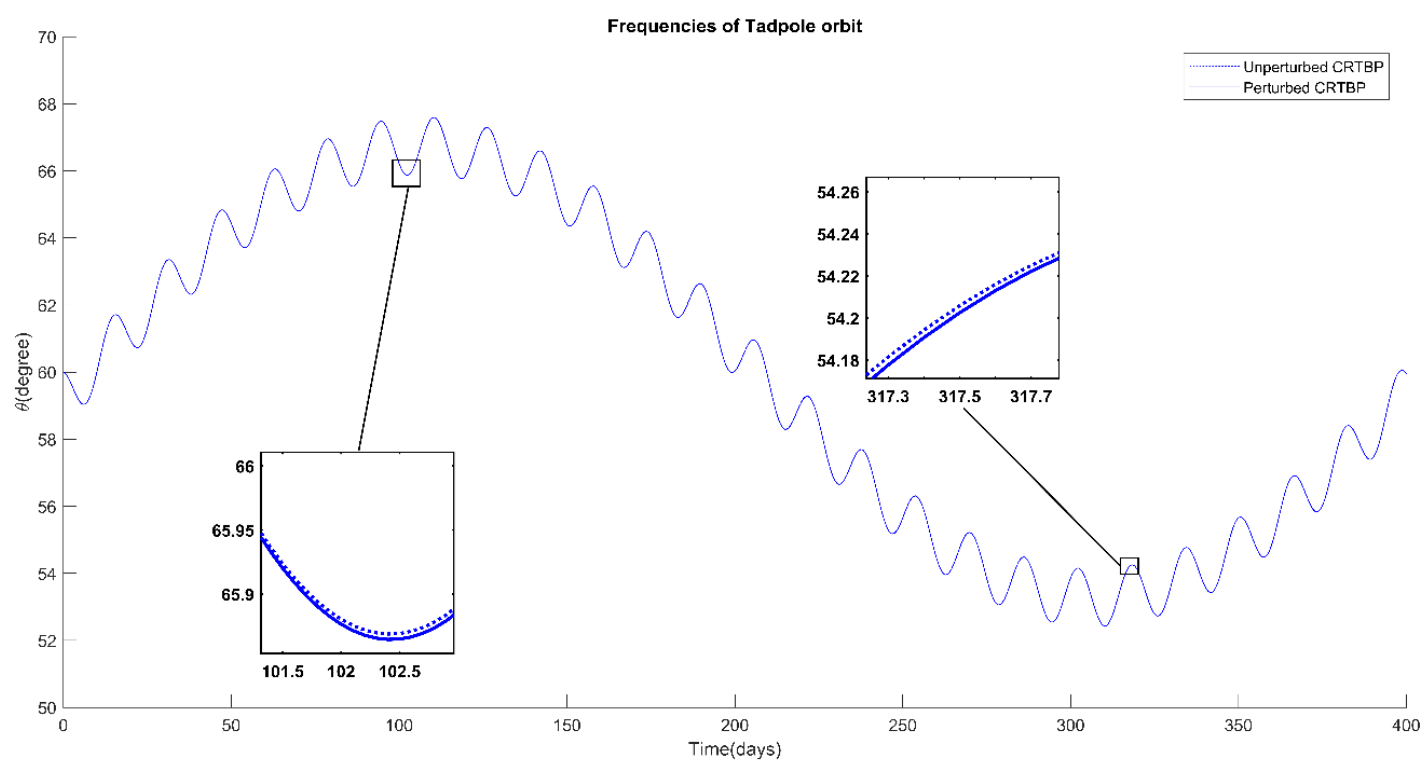

Fig 8. Frequencies of the Tadpole Orbits (true anomaly)

\section{Three-Dimensional Case}

The equations of motion in the three-dimensional case are ${ }^{(10)}$

$$
\begin{aligned}
& x^{\prime \prime}-2 n y^{\prime}=\Omega_{x}, \\
& y^{\prime \prime}+2 n x^{\prime}=\Omega_{y}, \\
& z^{\prime \prime}=\Omega_{z},
\end{aligned}
$$

where

$$
\Omega=\frac{n^{2}}{2}\left(x^{2}+y^{2}\right)+\left[\frac{1-\mu}{r_{1}}+\frac{\mu}{r_{2}}+\frac{(1-\mu) A_{1}}{2 r_{1}^{3}}-\frac{3(1-\mu) A_{1} z^{2}}{2 r_{1}^{5}}\right]
$$

With

$$
\begin{aligned}
& r_{1}^{2}=(x-\mu)^{2}+y^{2}+z^{2}, \\
& r_{2}^{2}=(x+1-\mu)^{2}+y^{2}+z^{2} .
\end{aligned}
$$

Similar to the planar RTBP, in the three-dimensional case also, the singularities are obtained by equating $x^{\prime}, y^{\prime}, z^{\prime}$ and hence $\Omega_{x}, \Omega_{y}, \Omega_{z}$ to zero. It can be proved that the system gets five equilibrium points in the $x y$-plane and the points are located in the same places as in the planar case ${ }^{(10)}$. At the equilibrium points, $\Omega_{x z}=\Omega_{y z}=0$, hence, the variational equations are given by ${ }^{(10)}$

$$
\begin{aligned}
& \xi^{\prime \prime}-2 n \eta^{\prime}=\Omega_{x x}(a, b, c) \xi+\Omega_{x y}(a, b, c) \eta \\
& \eta^{\prime \prime}+2 n \xi^{\prime}=\Omega_{x y}(a, b, c) \xi+\Omega_{y y}(a, b, c) \eta \\
& \zeta^{\prime \prime}=\Omega_{z z}(a, b, c) \zeta .
\end{aligned}
$$


where, $x=a+\xi, y=b+\eta, z=c+\zeta$. The second derivatives are to be evaluated at the equilibrium points. The motion, therefore, in the xy-plane does not influence the motion in the z-direction at the five equilibrium points. The mean in the $z$-direction is also affected by the second derivatives, whose relation at the collinear points is

$\Omega_{x x}+\Omega_{y y}+\Omega_{z z}=2 \mathrm{n}^{2}$.

The mean motion in the $\mathrm{z}$-direction is

$$
\begin{gathered}
s_{z}=\sqrt{-\Omega_{z z}}=\sqrt{\Omega_{x x}+\Omega_{y y}-2 n^{2}}, \\
s_{z}=1+\frac{3 A_{1}}{2}(3-\mu)>n . \\
s_{z}=1+\frac{3 A_{1}}{2}(3 / 2-\mu)>n .
\end{gathered}
$$

Equation (50) gives the angular velocities $s_{z}$ for the triangular points $\mathrm{L}_{4,5}$. This value is always greater than the mean motion of the primaries ${ }^{(10)}$ as observed from [ Table 8 ] and Equation (51). Equation (52) provides $\mathrm{s}_{z}$ when the mean motion is $\mathrm{n}^{2}=1+$ $3 A_{1} / 2$. From Equations (51) and (52), we easily notice that $s_{z}$ increases further with the new mean motion. This result will play an important role in studying three-dimensional periodic orbits around the triangular points with oblateness.

\section{Results}

The oblateness of the more massive primary is known to increase the mean motion of the primaries. With the secular perturbations included on argument of perigee $(\omega)$ and right ascension of ascending node $(\Omega)$ in the expression of mean motion, it is seen to further increase. With the new mean motion expression, the locations of the five equilibrium points of the circular restricted three-body problem are studied. The collinear equilibrium points move towards the more massive primary for all the three points, which is a new result. As opposed to this, the triangular points shift away from the origin in the $x$ direction due to oblateness. And, the $x$-location of the triangular points are found to be unaffected by the change in mean motion. However, the $y$-location is significantly affected by the change in mean motion. The points shift towards the origin in the $y$-direction and decrease by a high coefficient in the linear term of $A_{1}$. In the stability of the collinear points, the effect of oblateness on the eccentricities of the periodic elliptic orbits around the collinear points in conditional stability is changed by the new mean motion expression. The eccentricities for the points $L_{1}$ and $L_{3}$ increase but that for $L_{2}$ is decreases by the changed mean motion. This is a new result. The critical mass ratio for the triangular points are obtained. The critical mass ratio shows a further significant decrease due to the oblateness with new mean motion. Stability of the triangular equilibrium points for various cases of astronomical interest are studied. Series expansion of the angular frequencies till the linear terms suggest that both $s_{4}$ and $s_{5}$ increase with oblateness. $s_{4}$ shows further increase in its value due to new mean motion. The eccentricities of the retrograde periodic orbits around $L_{4,5}$ when $\mu=\mu_{c}$ are found. Till linear terms of $A_{1}$, the eccentricity is not affected by the change in mean motion; but an increase is observed when higher order terms in $A_{1}$ are considered. The tadpole orbits of the Saturn-Titan system get an increase in their time period of the short-periodic orbit and a decrease in the period of the long-periodic orbit. The study about the three-dimensional case of the equilibrium points brings out that the mean motion $s_{z}$ of triangular points in the $z$-direction increases further significantly with the new mean motion.

\section{Conclusion}

The CRTBP with the more massive primary as an oblate spheroid with its equatorial plane coincident with the plane of motion is studied. A new mean motion expression for this study is used which is obtained by using the secular perturbation effects of oblateness of the more massive primary on the mean anomaly, argument of perigee and the right ascension of the ascending node ${ }^{(1)}$. This increases the value of mean motion of the primaries. With this mean motion value, the locations of the five equilibrium points in the planar problem are found. The shifts in their locations due to the oblateness effect are studied. It is interesting to note that all the three collinear points move towards the more massive primary with oblateness. It is a new result. Among the shifts in the collinear points, $L_{3}$ shows the highest shift towards the more massive primary. And, when the shifts of all five equilibrium points are compared, the $y$-coordinate of the triangular point is the highest. The linear stability of the collinear equilibrium points is studied. It is very interesting to note that the eccentricities (e) of the orbits around $L_{1}$ and $L_{3}$ increase, while it decreases around $L_{2}$ with the addition of oblateness with the new mean motion. The decrease in e is significant 
in Saturn-Mimas system from 0.95036 to 0.87558 . For the triangular equilibrium points, the critical mass ratio $\mu_{c}$ within which the system remains linearly stable is calculated in terms of the oblateness coefficient. The effect of the change in mean motion value is clearly seen in the expression for critical mass ratio. $\mu_{c}$ further decreases significantly from $0.285 \ldots A_{1}$ to $0.365 \ldots A_{1}$ with the new mean motion. Linear stability of the triangular equilibrium points when the mass ratio is within the limit and when it equates to the critical mass ratio $\mu_{c}$ are studied. The periodic retrograde motion around the triangular equilibrium points in both of the cases are studied. The combined long- and short-periodic orbits around the Saturn-Titan system are studied. The study is extended to the three-dimensional case and the mean motion sz in the $z$-direction for the triangular points is calculated. The mean motion $S_{z}$ increases significantly with the new mean motion from $9 \mathrm{~A}_{1} / 4$ to $9 \mathrm{~A}_{1} / 2$.

\section{Acknowledgements}

The authors are highly thankful to the referees for their constructive comments and suggestions which has helped us to bring out the manuscript in the present form. Authors are also thankful to the Chief Editor for his kind interest of this study.

\section{References}

1) Sharma RK, Sellamuthu H, Jency AA. Perturbed Trojan dynamics in the solar system. In: AAS AIAA Astrodynamics Specialist Conference. 2020;p. 20-704. Available from: https://www.researchgate.net/publication/343547691.

2) Johnson A, Sharma RK. Locations of Lagrangian points and periodic orbits around triangular points in the photo gravitational elliptic restricted three-body problem with oblateness. International Journal of Advanced Astronomy. 2019;7:25-38. Available from: https://doi.org/10.14419/ijaa.v7i2.29377.

3) Jency AA, Sharma RK. Location and stability of the triangular Lagrange points in photo-gravitational elliptic restricted three body problem with the more massive primary as an oblate spheroid. International Journal of Advanced Astronomy. 2019;7(2). Available from: https://dx.doi.org/10.14419/ijaa.v7i2. 29814.

4) Arohan R, Sharma RK. Periodic orbits in the planar restricted photo-gravitational problem when the smaller primary is an oblate spheroid. Indian Journal of Science and Technology. 2020;13(16):1630-1640. Available from: https://doi.org/10.17485/IJST/v13i16.401.

5) Sharma RK. Perturbations of Lagrangian points in the restricted three-body problem. Indian Journal of Pure and Applied Mathematics. 1975;6:1099-1102.

6) Sharma RK, Rao PVS. Collinear equilibria and their characteristic exponents in the restricted three-body problem when the primaries are oblate spheroids. Celestial Mechanics. 1975;12(2):189-201. Available from: https://dx.doi.org/10.1007/bf01230211.

7) McCuskey SW. Introduction to Celestial Mechanics. and others, editor;Addison-Wesley Publishing Company. 1963.

8) Rao PVS, Sharma RK. A note on the stability of the triangular points of equilibrium in the restricted three-body problem. Astronomy and Astrophysics. 1975;43:381-383. Available from: http://adsabs.harvard.edu/abs/1975A\%26A....43..381S.

9) Sharma RK. Study of Periodic Orbits in Restricted Problem taking the Bigger Primary an Oblate Spheroid. Ph.D Thesis, University of Delhi. 1976.

10) Sharma RK, Rao PVS. Stationary solutions and their characteristic exponents in the restricted three-body problem when the more massive primary is an oblate spheroid. Celestial Mechanics. 1976;13(2):137-149. Available from: https://dx.doi.org/10.1007/bf01232721.

11) Sharma RK, Rao PVS. A case of commensurability induced by oblateness. Celestial Mechanics. 1978;18(2):185-194. Available from: https://dx.doi.org/ $10.1007 / \mathrm{bf01228715.}$

12) Sharma RK, Rao PVS. Effect of oblateness on triangular solutions at critical mass. Astrophysics and Space Science. 1979;60(2):247-250. Available from: https://dx.doi.org/10.1007/bf00644329.

13) Sharma RK. Periodic orbits of second kind in the restricted three-body problem when the more massive primary is an oblate spheroid. Astrophys And Space Science. 1981;76:255-258. Available from: https://doi.org/10.1007/.

14) Sharma RK. On linear stability of triangular libration points of the photogravitational three-body problem when the more massive primary is an oblate spheroid. In: Fricke W, Teleki G, editors. Sun and Planetary System. Dordrecht, Holland. D. Reidel Publishing Co. 1982;p. 435-436. Available from: https://doi.org/10.1007/978-94-009-7846-1_114.

15) Sharma RK, Rao PVS. On finite periodic orbits around the equilateral solutions of the planar restricted three-body problem. In: Bhatnagar KB, et al., editors. International workshop on Space Dynamics and Celestial mechanics. D. Reidel Publishing Co. 1986;p. 71-85. Available from: https: //doi.org/10.1007/978-94-009-4732-0_8.

16) Sharma RK. The linear stability of libration points of the photogravitational restricted three-body problem when the smaller primary is an oblate spheroid. Astrophys Space science. 1987;135:271-281. Available from: https://doi.org/10.1007/BF00641562.

17) Rao PVS, Sharma RK. Oblateness effect on finite periodic orbits at L4. In: and others, editor. 39th Congress of the International Astronautical Federation;vol. 300. . Available from: https://ui.adsabs.harvard.edu/\#abs/1988bang.iafcU....S/abstract.

18) Sharma RK. The periodic orbits of the second kind in terms of Giacaglia's variables with oblateness. Earth, Moon and Planets. 1989;45:312-318. Available from: https://ui.adsabs.harvard.edu/link_gateway/1989EM\&P...45..213S/doi:10.1007/BF00057744.

19) Sharma RK. Periodic orbits of third kind in the restricted three-body problem with oblateness. Astrophys And Space Science. 1990;166:211-218. Available from: https://doi.org/10.1007/BF01094894.

20) Rao PVS, Sharma RK. Effect of oblateness on the non-linear stability of L 4 in the restricted three-body problem. Celestial Mechanics and Dynamical Astronomy. 1997;65(3):291-312. Available from: https://dx.doi.org/10.1007/bf00053510.

21) Markellos VV, Papadakis KE, Perdios EA. Non-linear stability zones around triangular equilibria in the plane circular restricted three-body problem with oblateness. Astrophysics and Space Science. 1996;245(1):157-164. Available from: https://dx.doi.org/10.1007/bf00637811.

22) Jain S, Kumar A, Bhatnagar KB. Periodic orbits around the collinear liberation points in the restricted three body problem when the smaller primary is a triaxial rigid body: Sun-Earth case. Bull Astr Soc India. 2006;34:211-223. Available from: https://ui.adsabs.harvard.edu/\#abs/2006BASI...34..211J/abstract.

23) Shankaran S, Sharma JP, Ishwar B. Equilibrium points in the generalised photogravitational non-planar restricted three body problem. International Journal of Engineering, Science and Technology. 2011;3(2):63-67. Available from: https://dx.doi.org/10.4314/ijest.v3i2.68133.

24) Beevi AS, Sharma RK. Oblateness effect of Saturn on periodic orbits in the Saturn-Titan restricted three-body problem. Astrophysics and Space Science. 2012;340(2):245-261. Available from: https://dx.doi.org/10.1007/s10509-012-1052-3. 
25) Arredondo JA, Guo J, Stoica C, Tamayo C. On the restricted three body problem with oblate primaries. Astrophysics and Space Science. 2012;341(2):315322. Available from: https://dx.doi.org/10.1007/s10509-012-1085-7.

26) Abouelmagd EI, Asiri HM, Sharaf MA. The effect of oblateness in the perturbed restricted three-body problem. Meccanica. 2013;48(10):2479-2490. Available from: https://dx.doi.org/10.1007/s11012-013-9762-3.

27) Tiwary RD, Kushvah BS. Computation of halo orbits in the photogravitational Sun-Earth system with oblateness. Astrophysics and Space Science. 2015;357(1):1-16. Available from: https://dx.doi.org/10.1007/s10509-015-2243-5.

28) Pathak N, Thomas VO. Analysis of Effect of Oblateness of Smaller Primary on the Evolution of Periodic Orbits. International Journal of Astronomy and Astrophysics. 2016;06(04):440-463. Available from: https://dx.doi.org/10.4236/ijaa.2016.64036.

29) Chidambararaj P, Sharma RK. Halo Orbits around Sun-Earth L1 in Photogravitational Restricted Three-Body Problem with Oblateness of Smaller Primary. International Journal of Astronomy and Astrophysics. 2016;06(03):293-311. Available from: https://dx.doi.org/10.4236/ijaa.2016.63025.

30) Ansari AA, Alam M. Dynamics in the circular restricted three body problem with perturbations. International Journal of Advanced Astronomy. 2017;5(1):19-19. Available from: https://dx.doi.org/10.14419/ijaa.v5i1.7102.

31) Beevi AS, Sharma RK. Effect of Oblateness of the More Massive Primary on Periodic Orbits in the Restricted Three-Body Problem. Advances in Astrophysics. 2017;2:12-24. Available from: https://dx.doi.org/10.22606/adap.2017.21003.

32) Pathak N, Thomas VO, Abouelmagd EI. The perturbed photogravitational restricted three-body problem: analysis of resonant periodic orbits. Discrete and continuous dynamical systems: Series S. 2019;12:849-875. Available from: https://doi.org/10.3934/dcdss.2019057.

33) Szebehely V. Theory Of Orbits. New York. Academic Press. 1967. Available from: https://doi.org/10.1007/978-3-7091-2916-6_2.

34) Danby JMA. Fundamentals of Celestial Mechanics. 2nd ed. VA, USA. Willmann-Bell, Inc. 1988.

35) Murray CD, Dermott SF. Solar System Dynamics. Cambridge. Cambridge University Press. 1999.

36) Rabe E. Determination and survey of periodic Trojan orbits in the restricted problem of three bodies. The Astronomical Journal. 1961;66(9):500-500. Available from: https://dx.doi.org/10.1086/108451.

37) Singh J, Ishwar B. Stability of triangular points in the generalised photogravitational restricted three body problem. Astronomical Society of India. 1999;27:415-424. Available from: http://adsabs.harvard.edu/abs/1999BASI...27..415S. 